\title{
Optics
}

2012; 1(1) : 1-10

Published online December 30, 2012 (http:// www.sciencepublishinggroup.com/j/optics)

doi: $10.11648 /$ j.optics.20120101.11

\section{Planar gradient hyperbolic secant lens for subwavelength focusing and superresolution imaging}

\author{
V. V. Kotlyar, A. A. Kovalev*, A. G. Nalimov \\ Laser Measurements Laboratory of the Image Processing Systems Institute of the Russian Academy of Sciences, 151 Molodogvardeiskaya \\ street, Samara, Russia
}

\section{Email address:}

kotlyar@smr.ru (V. V. Kotlyar), alexeysmr@smr.ru (A. A. Kovalev), anton@smr.ru (A. G. Nalimov)

\section{To cite this article:}

V. V. Kotlyar, A. A. Kovalev, A. G. Nalimov. Planar Gradient Hyperbolic Secant Lens for Subwavelength Focusing and Superresolution Imaging. Optics. Vol. 1, No. 1, 2012, pp. 1-10. doi: 10.11648/j.optics.20120101.11

\begin{abstract}
Integral relations to describe the propagation of a TE-wave from an external point source through the two-dimensional medium (plane interface) and the plane-parallel plate are proposed. We discuss three types of waves that contribute to the resulting light field, namely, the propagating waves and the first- and second-type surface waves. The comparison of near-field refractive lenses (SIL, NAIL) and a planar hyperbolic secant lens shows their numerical apertures to have close values, with the difference being as small as 5\% for the Si-based optical elements. The FDTD-method simulation shows that by combining the gradient-index hyperbolic secant lens with a subwavelength diffraction grating or replacing it with its binary analog, the focal spot size can be made, respectively, $10 \%$ and $20 \%$ smaller than the diffraction-limited resolution in the 2D medium. We design a Si-based, planar binary microlens to generate a near-surface focal spot of full-width half-maximum size FWHM $=0.102 \lambda$, where $\lambda$ is the incident wavelength, which is practically devoid of side-lobes. It is shown that about 10 percent of the total incident beam energy goes to the far-field zone.
\end{abstract}

Keywords: Superresolution, Gradient-Index Lens, Secant Lens, Near-Field Lenses

\section{Introduction}

The combined use of diffractive optical elements (DOE) and far-field focusing refractive optics makes it possible to achieve the superresolution, generating a focal spot of full-width half-maximum size FWHM $=0.44 \lambda[1]$, where $\lambda$ is the wavelength of light in vacuum, thus breaking the diffraction limit of FWHM $=0.51 \lambda$. Note, however, that the side-lobes of the resulting diffraction pattern will amount to $20 \%$ of the focal spot central peak. Although it is possible to obtain even smaller size focal spots for the far-field diffraction pattern but the proportion of light energy going to the side-lobes will accordingly increase and can eventually become comparable with or equal to the central focal spot intensity [2]. Note that there are several definitions of the diffraction-limited resolution in optics: the Rayleigh resolution limit of $0.61 \lambda / N A$ [3], the Houston limit of $0.5 \lambda / N A$, and the Sparrow limit of $0.475 \lambda / N A$ [4], where $N A$ is the numerical aperture of the focusing system. For the purpose of this study, the resolution limit is defined for the 3D fields as the full-width half-maximum intensity of the Airy function squared $(0.51 \lambda / N A)$ and for the $2 \mathrm{D}$ fields as the full-width half-maximum intensity of the sinc-function $(0.44 \lambda / N A)$.
To overcome the resolution limit without increasing the side-lobes the optical element should be put closer to the light source. This area of optics is dealt with using near-field microscopy techniques [5]. Eliminating from consideration the metallic surfaces and surface plasmons [6], in which a superresolution of $\lambda / 50$ is attained due to a larger real part of the metal permittivity, and staying within the framework of refractive and gradient optics, the enhanced resolution can be achieved with the aid of near-field lenses, such as SIL (solid immersion lens) [7], NAIL (numerical aperture immersion lens) [8-10] and nSIL (nano solid immersion lens) $[3,11]$.

Using a combination of the SIL (for wavelength $\lambda=633$ $\mathrm{nm}$ ) and a glass hemisphere LASFN9 of radius $5 \mathrm{~mm}$ and refractive index $n=1.845$, the superresolution of FWHM $=$ $190 \mathrm{~nm}=0.298 \lambda$ has been achieved experimentally [7]. Using a Si hemisphere and a NAIL of radius $1.6 \mathrm{~mm}$, the superresolution of FWHM $=250 \mathrm{~nm}=0.23 \lambda$ was experimentally achieved (for $\mathrm{Si}$, the theoretical diffraction limit is $\mathrm{FWHM}=0.147 \lambda$ at $n=3.4$ and $\lambda=1 \mu \mathrm{m}$ ) in Ref. [8]. In a later work [9], the achievement of a superresolution of $\mathrm{FWHM}=145 \mathrm{~nm}=0.11 \lambda(\lambda=1.3 \mu \mathrm{m})$ by means of the annular aperture and a Si NAIL was reported. If the lens is 
illuminated by an annular beam, the focal spot is formed by the Bessel beam, for which the resolution limit is FWHM = $0.36 \lambda / N A$. It has been experimentally demonstrated [3] that using the near-field optics (nSIL) and a glass hemisphere of radius $1-2 \mu \mathrm{m}(n=1.6)$ a focal spot of diameter FWHM $=$ $126 \mathrm{~nm}=0.235 \lambda(\lambda=532 \mathrm{~nm})$ can be obtained [11]. In Ref. [12] a subwavelength focal spot of diameter FWHM $=0.31 \lambda$ was realized using a helical phase plate and a SIL with $N A=$ 1.7. It should be noted that the near-field refractive optics is able to enhance the numerical aperture of the already converging beam, whereas the focusing of the beam propagating from a point source calls for the use of an extra optical component.

The near-field focusing can be done using a single optical microelement. For example, in Ref. [13] a microdisc with $400-\mathrm{nm}$ height and diameter $10-\mu \mathrm{m}$ was used to experimentally obtain a focal spot of diameter FWHM $=0.86 \lambda$. In Refs. [14] and [15] experiments with planar gradient Luneburg microlenses were described, although the focal spot diameters reported in the experiments were not record-breaking. An image with a resolution of FWHM = $0.43 \lambda$ with use of a planar photonic crystal was formed in Ref. [16]. Note that a near-field image can also be obtained with use of a superlens, FWHM $=0.28 \lambda$ [17], and a hyperlens, FWHM $=0.38 \lambda$ [18]. In Ref. [19] it was numerically shown that a spiral plasmonic lens of diameter $8 \mu \mathrm{m}$ allows the plasmon to be focused into a spot of size FWHM $=0.35 \lambda$. A 3D plasmonic superlens with a sinusoidal grating was designed in Ref. [20], allowing two lines of width $0.05 \lambda$ spaced $0.2 \lambda$ apart to be resolved. In Refs. [21, 22] the focal spots were experimentally obtained on the tip of dielectric and metallic microcones.

Superoscillation-based (SOL) imaging and focusing of light in the far-field zone was considered in Ref. [2] (FWHM $=0.17 \lambda)$ and Ref. [23] $(\mathrm{FWHM}=0.36 \lambda)$. In Ref. [24] a focal spot of size $\mathrm{FWHM}=0.58 \lambda=0.39 \lambda / \mathrm{NA}$ was generated experimentally $1-\mu \mathrm{m}$ apart from the binary microaxicon. In Ref. [25], a radially polarized beam was focused into a spot of diameter FWHM $=0.33 \lambda$ using an annular diaphragm and a microobjective with $\mathrm{NA}=1.4$.

There are also works where the superresolution has been achieved by the refinement of traditional optical elements and systems. In Ref. [26] with use of a cardioid annular condenser $(\mathrm{NA}=1.4)$ a Richardson slide pattern image was obtained for coherent light with a $90-\mathrm{nm}$ resolution $(0.2 \lambda)$. In Ref. [27] using the solid-immersion imaging interferometric nanoscopy a possibility was shown to achieve a resolution equal to the diffraction limit in the medium, FWHM $=\lambda /(2 n)$, where $n$ is the medium refractive index.

From the overview above it is seen that the best resolution has been achieved with use of SIL microscopy (FWHM = $0.11 \lambda)$ [9] and SOL microscopy $(\mathrm{FWHM}=0.17 \lambda)[2]$.

In this paper, we design a planar binary near-field dielectric lens which allows obtaining a focal spot of size FWHM $=0.102 \lambda$. We also describe a mechanism by which the superresolution is achieved using the near-field optics, including a gradient-index planar lens. We show that inho- mogeneous evanescent waves from a point source (first-type surface waves, $k<k_{x}<n k$, where $k$ and $k_{x}$ are the wavenumber in vacuum and the $x$-projection of the wavevector, respectively, $n$ is the refractive index of the medium) are partially tunneled into the medium (lens) and transformed into the medium modes, thus contributing to the source image alongside with the conventional propagating waves $\left(0<k_{x}<k\right)$. In the far-field optics, the first-type waves fail to achieve the image plane. The second-type surface waves $\left(k_{x}>\right.$ $n k)$ transformed into the medium surface waves are propagating along the lens input plane, so that only their exponentially damped tails achieve the lens output (image) surface.

We derive integral relations to describe the propagation of a TE-wave in the 2D medium from an external light source (with the plane interface) and similar relations to describe the light field behind a plane-parallel plate. The comparison of the numerical apertures of the near-field refractive lenses (SIL, NAIL) and a planar hyperbolic secant lens shows them to have similar values, with the difference being equal to $5 \%$ for Si-based elements. The simulation using the FullWAVE software shows that by combining the gradient-index hyperbolic secant lens with a subwavelength diffraction grating or replacing it by a binary analog the focal spot size can, respectively, be made $10 \%$ and $20 \%$ smaller than the diffraction limit in the given medium.

\section{Tunneling of Inhomogeneous Waves from the Source into the Medium}

For the 2D case, the electric field strength of a monochromatic TE-wave at distance $\mathrm{z}$ from the initial plane takes the form (formula of decomposition of field into angular spectrum of plane waves [28]):

$$
\begin{aligned}
& E_{1}(x, z)=\frac{k}{2 \pi} \int_{-\infty}^{+\infty} \int_{-\infty}^{+\infty} E_{0}\left(x^{\prime}, z=0\right) \times \\
& \times \exp \left[-\mathrm{i} k \xi\left(x^{\prime}-x\right)+\mathrm{i} k z \sqrt{1-\xi^{2}}\right] \mathrm{d} x^{\prime} \mathrm{d} \xi
\end{aligned}
$$

where $\xi=k_{x} / k$.

A point source in the initial plane:

$$
E_{0}(x, z=0)=E_{0} \delta\left(\frac{k x}{2 \pi}\right),
$$

where $\delta(x)$ is the Dirac delta-function, produces at distance $z$ the field amplitude equal to the sum of plane waves and inhomogeneous evanescent waves:

$$
E_{1}(x, z)=E_{0} \int_{-\infty}^{\infty} \exp \left[-\mathrm{i} k \xi x+\mathrm{i} k z \sqrt{1-\xi^{2}}\right] \mathrm{d} \xi
$$

In Eq. (3) at $|\quad|<1$ the propagating plane waves contribute into the field $\mathrm{E} 1(\mathrm{x}, \mathrm{z})$, while at $1<|\quad|<\infty$ the evanescent (surface) waves contribute into the field E1(x, z).

Note that since the Hankel function of zero order and first kind is given by [29] 


$$
H_{0}^{1}\left(k \sqrt{x^{2}+z^{2}}\right)=\frac{1}{\pi} \int_{-\infty}^{\infty} \frac{\exp \left[-\mathrm{i} k \xi x+\mathrm{i} k z \sqrt{1-\xi^{2}}\right]}{\sqrt{1-\xi^{2}}} \mathrm{~d} \xi,
$$

equation (3) can be expressed through the derivative of the Hankel function:

$$
E_{1}(x, z)=-\frac{\mathrm{i} \pi}{k} \frac{\partial}{\partial z} H_{0}^{1}\left(k \sqrt{x^{2}+z^{2}}\right) .
$$

If we put a medium interface at distance $z$ from the source, the light will pass into the medium with refractive index $n$. Then, the E-vector amplitude at distance $z$ from the source will be equal to

$$
E_{2}(x, z)=E_{0} \int_{-\infty}^{\infty} T_{1}(\xi) \exp \left[-\mathrm{i} k \xi x+\mathrm{i} k z \sqrt{n^{2}-\xi^{2}}\right] \mathrm{d} \xi
$$

where

$$
T_{1}(\xi)=\left\{\begin{array}{l}
\frac{2 \sqrt{1-\xi^{2}}}{\sqrt{1-\xi^{2}}+\sqrt{n^{2}-\xi^{2}}}, 0<|\xi|<1, \\
\frac{2 \mathrm{i} \sqrt{\xi^{2}-1}}{\mathrm{i} \sqrt{\xi^{2}-1}+\sqrt{n^{2}-\xi^{2}}}, 1<|\xi|<n, \\
\frac{2 \sqrt{\xi^{2}-1}}{\sqrt{\xi^{2}-1}+\sqrt{\xi^{2}-n^{2}}},|\xi|>n .
\end{array}\right.
$$

The values of $T_{1}(\xi)$ in equation (7) describe the coefficients derived from Fresnel formulae [30] for three different cases: conversion of the propagating plane wave into a propagating plane wave; conversion of the evanescent plane wave into a propagating plane wave; and conversion of the evanescent plane wave into an evanescent plane wave in the medium.

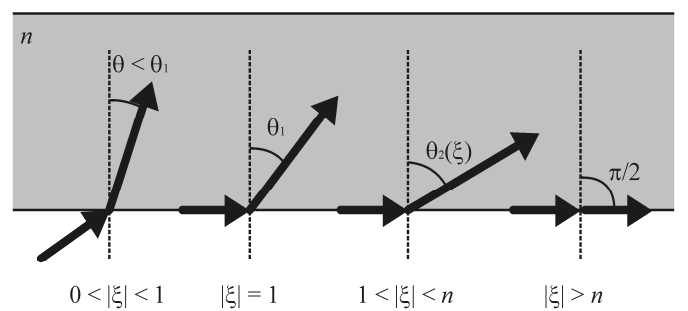

Figure 1. Different types of waves tunneling into the media with refractive index $n$.

Actually, from equation (7) it is seen that the propagating waves from a point source in the medium with $n=1$ and $0<$ $|\xi|<1$, where $\xi=k_{x} / k$, entering the second medium at angles $0<\theta<\theta_{1}$, where $\theta_{1}=\arcsin (1 / n)$, will then propagate in the medium with $n>1$ (figure 1). The first-type surface waves from the source that have the wavenumber projection in the interval $1<|\xi|<n$ will enter the second medium at angles $\theta_{2}(\xi)=\arcsin (\xi / n)$ found in the range $\theta_{1}<\theta_{2}(\xi)<$ $\pi / 2$, because the maximal angle $\theta_{2}(\xi)$ equals $\pi / 2$ at $\xi=n$. Being converted from the surface evanescent into the propagating, these waves will further propagate in the me- dium with $n>1$. The second-type surface waves from the source with $|\xi| n$ will represent the surface waves of the medium, propagating along the medium interface.

\section{Numerical Aperture of a Hyperbolic Secant Lens}

Let us consider a 2D hyperbolic secant (HS) lens whose refractive index is given by $[31,32]$

$$
n(x)=\frac{n}{\cosh \left(\frac{\pi x}{2 L}\right)}
$$

where $n$ is the refractive index on the lens axis, $L$ is the lens length, and $x$ is the coordinate in the transverse plane. If the lens (8) is of length $L$, all the rays parallel to the optical axis are intersected in the focal point at distance $L$. If the lens is of length $2 L$, the on-axis point source is imaged at distance $2 L$.

The numerical aperture of the HS lens can be derived from the ray equation in the gradient-index medium: $n(x) \cos \theta(x)=$ const, where $\theta(x)$ is the angle between the tangent to the ray and the optical axis $z$. Assume that the ray is incident on the lens in parallel with and at distance $x=R$ from the optical axis, where $R$ is the HS lens radius, which can be derived from the condition $n(R)=1: R=\operatorname{arccosh}(n)$. Then, the ray equation takes the form: $n \cos \theta_{0}=$ $n(R) \cos \theta(R)=1$, where $\theta_{0}$ is the angle between the tangent to the ray and the optical axis $z$ at the point of intersection of the ray and the optical axis. Whence it follows that $\cos \theta_{0}=$ $1 / n$, i.e. the HS lens numerical aperture is $N A=n \sin \theta_{0}=$ $\left(n^{2}-1\right)^{1 / 2}$, where $\theta_{0}=\arcsin \left[\left(n^{2}-1\right)^{1 / 2} / n\right]$. Then, the plane waves propagating at angles $\theta_{1}$ and $\theta_{2}$ smaller than $\theta_{0}$ will contribute to the focal spot at the HS lens output. Let us find the maximal $\xi_{\max }$ for the light waves contributing to the HS-lens-aided focal spot. From the equality $\theta_{2}\left(\xi_{\max }\right)=0$, we obtain $\arcsin (\xi / n)=\arcsin \left[\left(n^{2}-1\right)^{1 / 2} / n\right]$. From this equality it follows that $\xi_{\max }=\left(n^{2}-1\right)^{1 / 2}$. For silicon and wavelength $\lambda$ $=1550 \mathrm{~nm}$, we obtain $\xi_{\max }=3.32$, because $n=3.47$. Then, at the planar HS lens output, the focal spot full-width half-maximum size is $(n=3.47)$ :

$$
F W H M=\frac{0.44 \lambda}{N A}=\frac{0.44 \lambda}{\sqrt{n^{2}-1}}=\frac{0.44 \lambda}{\xi_{\max }}=0.133 \lambda .
$$

The theoretical resolution limit that can be derived using the near-field planar optics, such as SILs [7] and NAILs [8] equals $(n=3.47)$ :

$$
F W H M=0.44 \frac{\lambda}{n}=0.127 \lambda .
$$

Equation (10) stems from the fact that the numerical aperture of the SIL and NAIL, $N A_{S L L}=n \sin \theta \leq N A_{N A I L}=$ $\left(n^{2}-\cos ^{2} \theta\right)^{1 / 2}$ tends to $N A_{\max }=n$ in the limit $(\theta \rightarrow \pi / 2)$. The diffraction-limited focal spot of equation (10) is only $5 \%$ smaller than the focal spot produced by the HS lens, equation (9). Let us estimate the maximal angle at which the rays 
are propagating within the HS lens. The propagating plane waves from the source with the relative wavenumber projection found in the interval $0<|\xi|<1$ are transformed in the isotropic medium $(n=3.47)$ into the propagating waves traveling at angles found in the interval $0<\theta<\theta_{1}=\arcsin (1 / n) \cong 17^{0}$, with the maximal angle (to the optical axis) at which the rays can propagate in the HS lens being $\theta_{0}=\arcsin \left[\left(n^{2}-1\right)^{1 / 2} / n\right] \approx 74^{\circ}$.

Note that if a SIL or a NAIL is illuminated by an annular light beam the diffraction pattern in the focal plane will be described not by the Airy function (or the sinc-function for the $2 \mathrm{D}$ case) but by the zero-order Bessel function. Therefore, the theoretical resolution limit in the medium will be given by

$$
F W H M=0.36 \frac{\lambda}{n}=0.104 \lambda
$$

\section{Three Types of Waves Propagating in a Hyperbolic Secant Lens}

Note, however, that not all waves entering the HS lens will go outside. Assume that waves from the source enter a bounded (with respect to the optical axis $z$ ) medium in the form of a plane-parallel plate of thickness $d$. After passing the plate, the output waves will be described by the expression:

$$
E_{3}(x, z)=E_{0} \int_{-\infty}^{\infty} T_{2}(\xi) \exp \left[-\mathrm{i} k \xi x+\mathrm{i} k z \sqrt{1-\xi^{2}}\right] \mathrm{d} \xi
$$

where

$$
T_{2}(\xi)=\left\{\begin{array}{l}
\frac{2 \sqrt{\left(1-\xi^{2}\right)\left(n^{2}-\xi^{2}\right)}}{2 \sqrt{\left(1-\xi^{2}\right)\left(n^{2}-\xi^{2}\right)} \cos A-\mathrm{i}\left(n^{2}+1-2 \xi^{2}\right) \sin A}, \\
\text { where } 0<|\xi|<1, \\
\frac{2 \sqrt{\left(\xi^{2}-1\right)\left(n^{2}-\xi^{2}\right)}}{2 \sqrt{\left(\xi^{2}-1\right)\left(n^{2}-\xi^{2}\right)} \cos A-\left(n^{2}+1-2 \xi^{2}\right) \sin A}, \\
\text { where } 1<|\xi|<n, \\
\frac{2 \sqrt{\left(\xi^{2}-1\right)\left(\xi^{2}-n^{2}\right)}}{2 \sqrt{\left(\xi^{2}-1\right)\left(\xi^{2}-n^{2}\right)} \cosh B-\left(n^{2}+1-2 \xi^{2}\right) \sinh B}, \\
\text { where }|\xi|>n,
\end{array}\right.
$$

$A=i B=k d\left(n^{2}-\xi\right)^{1 / 2}$. Similarly to $T_{1}$ in equation (7), the magnitudes $T_{2}(\xi)$ represent the coefficients derived from the Fresnel formulae [30] for three different cases. Equation (13) suggests that after passing through the plate, the plane waves propagating from the source $(0<|\xi|<1)$ will propagate behind the plate at the same angles (since the medium prior and after the plate is air, $n=1)$. The first-type surface waves $(1<|\xi|<n)$ are converted into the modes within the plane-parallel plate and again into the surface waves on the opposite (relative to the source) plate surface. Thus, these waves will not propagate in the space behind the plate. With the second-type surface waves $(\mid \xi>n)$ being converted into the surface waves on the source-facing plate side, only their exponentially dumped "tails" reach the opposite plate side. Thus, the central rays from the source that propagate in the HS lens at angles to the optical axis smaller than $\theta_{1}=\arcsin (1 / n) \cong 17^{0}$ for $n=3.47$ can pass through and further propagate behind the lens with a length of $2 L$ in (8). The first-type surface waves from the source propagate in the HS lens as in a ring cavity, not going outside the lens. The second-type surface waves from the source remain the HS-lens surface waves, experiencing partial scattering from the lens's acute corners because the lens geometry is different from that of the plane-parallel plate, being limited by the transverse coordinates.

Thus, if we assume that the HS-lens-aided focal spot is generated only by the propagating waves with a maximal tilt of $\theta_{1}$, the expected focal spot size is $\left(n=3.47 ; \theta_{1}=17^{0}\right)$ $F W H M=0.44 \lambda /\left(n \sin \theta_{1}\right)=0.43 \lambda$. However, taking into consideration the essential contribution of the first-type surface waves propagating in the HS lens with a maximal tilt to the optical axis of $\theta_{0}=74^{0}$, the focal spot diameter is $F W H M=0.44 \lambda /\left(n \sin \theta_{0}\right)=0.132 \lambda$. This value is in good agreement with the simulation results [32] and with equation (9), which, in fact, leads to the same result, although in a different way.

Figure 2 presents a characteristic plot of the focal spot size against the plane-wave spectrum width contributing to the focus according to equation (9).

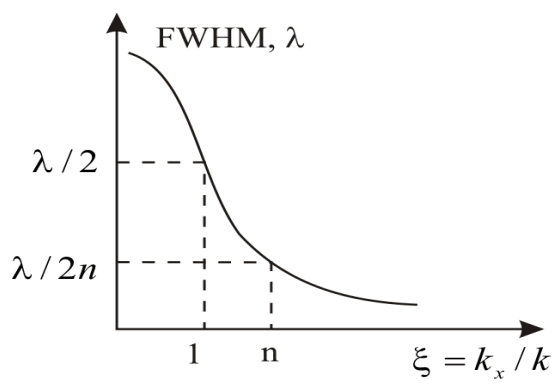

Figure 2. The point-source image diameter, equation (9), vs the plane-wave spectrum width, including the evanescent waves reaching the image plane.

Thus, if the simulation shows that the HS-lens-aided focal spot size is smaller than the diffraction-limited size in the 2D medium (equation (10)), we can infer that the second-type surface waves also contribute to the focal spot.

\section{Numerical Apertures of Near-Field Optics}

Below, we conduct the comparison of the $N A$ of the near-field SIL and NAIL lenses with the NA of the HS lens. The comparison results were briefly discussed in Section 2. Assume the propagation in free space of a converging light beam with $N A=\sin \theta$, where $\theta$ is the maximal angle which the beam rays make with the optical axis. If such a beam enters a medium with the refractive index $n$ and a plane interface, its numerical aperture will remain unchanged, $N A$ $=n \sin \beta=\sin \theta$, where $\beta$ is the maximal angle of the beam 
rays in the medium. The $N A$ of the initial beam can be enhanced using a SIL, which comprises a hemisphere with refractive index $n$, with its spherical surface placed in such a manner that the rays are incident normally (figure 3(a)). In this case, with the converging beam focused at the centre of the hemisphere, its $N A$ within the sphere will be defined by $N A_{S I L}=n \sin \theta$. Thus, using the near-field optics, with the focus found on the hemisphere flat surface, the $N A$ of the initial beam can be $n$ times enhanced or the focal spot diameter $n$ times reduced.

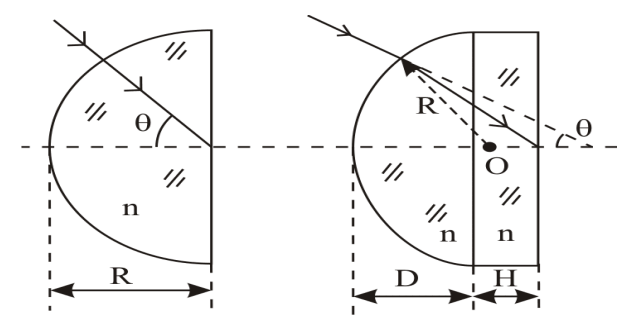

(a)

(b)

Figure 3. Diagram of incident and refracted rays for (a) SIL and (b) NAIL.

With another near-field lens - the NAIL, it becomes possible to achieve still higher values of the beam $N A$. In this case, a sphere segment (smaller than hemisphere) is put in the path of the converging beam. The rays should fall on the spherical surface in such a manner that their angle with the optical axis is smaller than the angle between the axis and the normal drawn to the spherical surface at the point of ray intersection. The optical arrangement should also contain a cylinder of the same radius as that of the sphere and of height $\mathrm{H}$, made of the same material. The cylinder should be fitted in such a manner that the rays are focused just on the intersection of its rear plane with the optical axis. Assume that the sphere's radius is $R$ and the segment height is $D<R$, then deriving the cylinder's height from the equation $D+H=$ $R(1+1 / n)$, we obtain the focal spot at the cylinder output plane (figure 3(b)). It is seen from figure 3(b) that the ray is refracted in the lens and falls on the optical axis at an angle larger than the angle $\theta$ of the ray incident on the lens. Considering that the $N A$ of the incident beam on the NAIL is $N A$ $=\sin \theta$, the $N A$ of the beam traveling in the cylinder and converging on the cylinder's output surface is $N A_{N A I L}=\left(n^{2}-\cos ^{2} \theta\right)^{1 / 2}$. It can be shown that $N A_{N A I L} \geq N A_{S I L}$. The comparison of the above NA values with the HS-lens's $N A=\left(n^{2}-1\right)^{1 / 2}=n \sin \theta_{0}$ shows that the difference is not large: the difference between the maximal $N A$ of the near-field refractive optics $N A_{N A I L}=N A_{S I L}=n=3.47$ and the $N A$ of the HS lens $N A=\left(n^{2}-1\right)^{1 / 2}=3.32$ is as small as $5 \%$. It should be, however, noted that the SIL and NAIL are only able to gather light from the source, converging the first-type surface waves into the propagating waves without focusing. Additional refractive optics should be employed to perform the subsequent focusing. On the contrary, the HS lens is capable of both gathering and focusing light on its output surface.

\section{Focal Spot Reduction by Modulation of Refractive Index}

In recent papers [33, 34], it was numerically demonstrated that using subwavelength gratings the surface waves from a point source could be converted into the propagating waves, thus achieving the superresolution of $\lambda / 20$. This was implemented by means of several stacked diffraction gratings of different subwavelength periods and arbitrary apertures [33] and a metallic subwavelength grating with high permittivity $(\varepsilon=-100)$ [34]. However, the use of focusing or imaging optical elements was not considered in the above works.

The simulation using the FullWAVE software (RSoft) implementing the finite-difference time-domain (FDTD) approach has shown that by combining a HS lens with a subwavelength grating or using a binary HS lens with subwavelength features the focal spot size can be, respectively, reduced by $10 \%$ and $20 \%$.

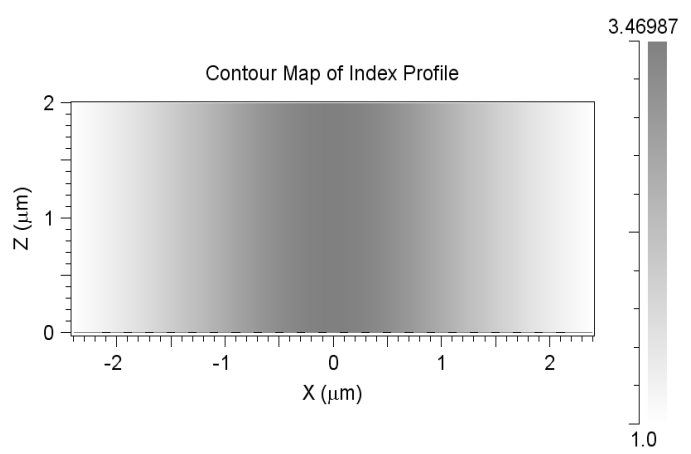

(a)

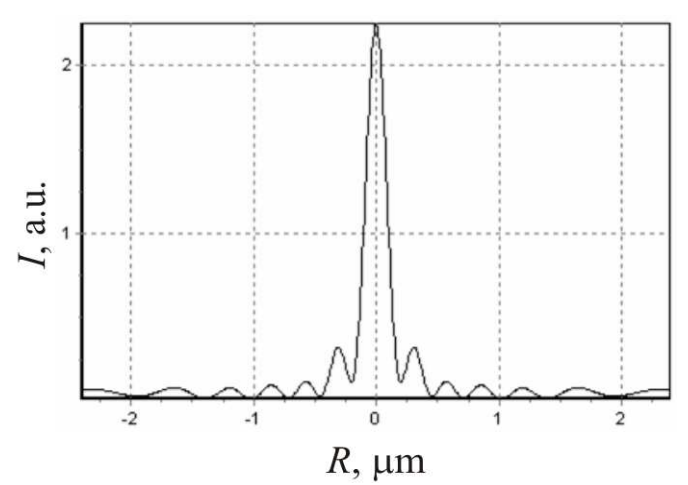

(b)

Figure 4. (a) Refractive index profile in the gradient-index HS lens (on the axis, $n=3.47$ ), horizontal dimension, $2 R=4.8 \mu \mathrm{m}$, vertical, $L=2 \mu \mathrm{m}$. Light propagates vertically. (b) Transverse intensity profile $\left|E_{y}\right|^{2}$ at the lens output (10 nm apart).

Figure 4 shows (a) the (gray-level) index profile of the HS lens (8) and (b) the intensity distribution at the lens output. The lens was illuminated by a plane TE-wave, i.e. electric field vector had only one component along Y-axis. The focal spot size in figure 4(b) is FWHM $=191 \mathrm{~nm}=0.123 \lambda, \lambda=$ 
$1.55 \mu \mathrm{m}$. This value is somewhat smaller than the diffraction limit in silicon, equation (10), thus proving that the second-type surface wave contribute to the HS-lens-aided focal spot.

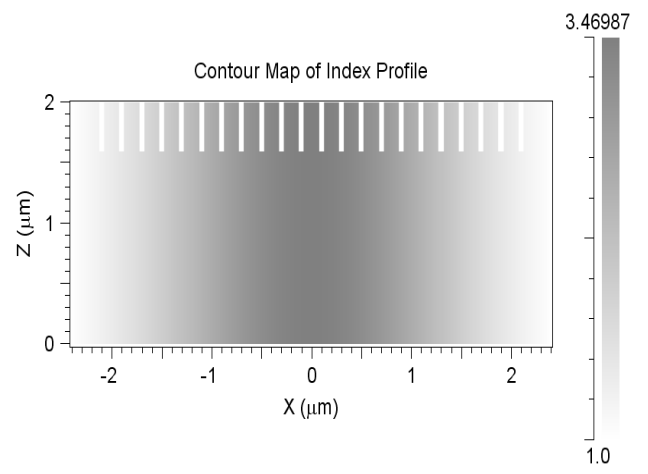

(a)

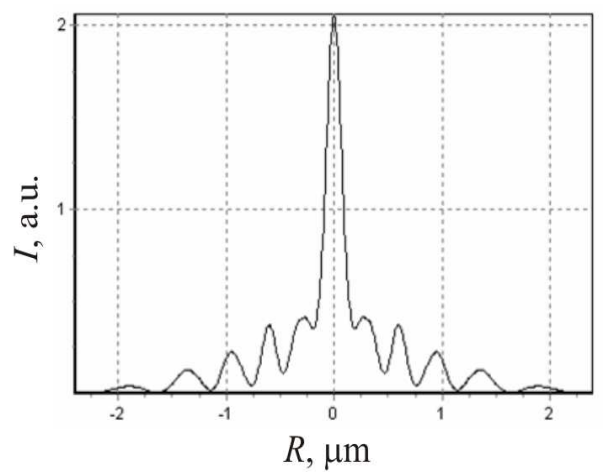

(b)

Figure 5. (a) Refractive index profile in the gradient-index HS lens ( $n=$ 3.47), horizontal dimension, $2 R=4.8 \mu \mathrm{m}$, vertical, $L=2 \mu \mathrm{m}$. On top of the $H S$ lens, there is a diffraction grating of depth $0.4 \mu \mathrm{m}$, period $0.2 \mu \mathrm{m}$, and groove width $0.05 \mu \mathrm{m}$ (the grooves filled with air). Light propagates from the bottom upwards. (b) Transverse intensity distribution $\left|E_{y}\right|^{2}$ at the lens output (10 $\mathrm{nm}$ apart).

Figure 5(a) depicts the same HS lens as in figure 4, but in combination with a subwavelength diffraction grating put on the lens top (output) surface and figure 5(b) shows the intensity distribution at the lens output. The focal spot size in figure $5(\mathrm{~b})$ is $\mathrm{FWHM}=177 \mathrm{~nm}=0.114 \lambda$. This is $8 \%$ smaller than the focal spot size in figure 4(b) and $10 \%$ smaller than the diffraction limit of equation (10).

\section{Amount of Energy in the Focal Spot}

Let us place in front of the lens (at distance $\lambda / 16 \approx 97 \mathrm{~nm}$ ) a source of a bounded, $2.4-\mu \mathrm{m}$ wide plane wave (i.e. the wave width is half the lens width). Some part of light will be reflected from the front surface of the lens, thus not affecting the focal spot. In order to determine the amount of the light energy that achieves the rear plane it is insufficient to measure the power flux through it, because some part of light is reflected from the rear plane and the total power flux of this part equals zero, although it affects the focal spot. Therefore, let us replace the lens with a secant waveguide, where all the light will propagate further instead of being reflected from the lens's rear plane. It appears that in this case $74.9 \%$ of the source power achieves the lens rear surface.

Assume that an infinite plane wave (i.e. of the width exceeding the simulation domain) is incident on the lens. Shown in figure 6 are the distributions of the energy density (i.e. intensity $\left|E_{y}\right|^{2}$, figure 6(a)) and the power flux density (i.e. Poynting vector, figure 6(b)) calculated in a plane located at distance $\lambda / 50 \approx 31 \mathrm{~nm}$ beyond the lens's rear plane. In figure 6(a), the width of the central peak is FWHM $=192$ $\mathrm{nm}=0.124 \lambda$, which is slightly smaller than the diffraction limit. In figure $6(\mathrm{~b})$, the width of central peak is FWHM = $213 \mathrm{~nm}(0.137 \lambda)$.

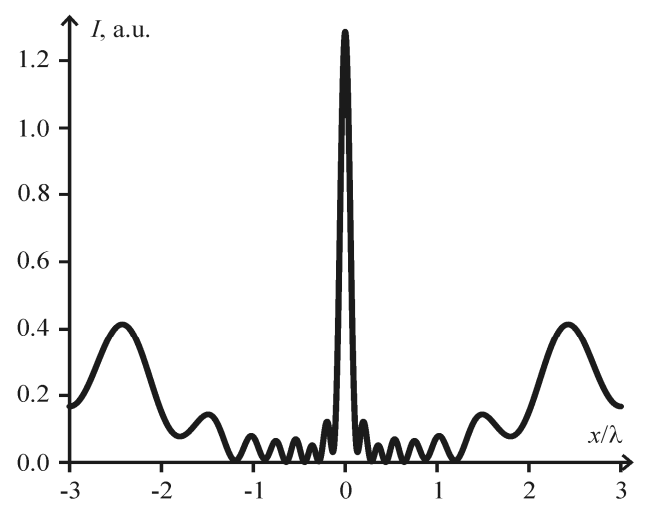

(a)

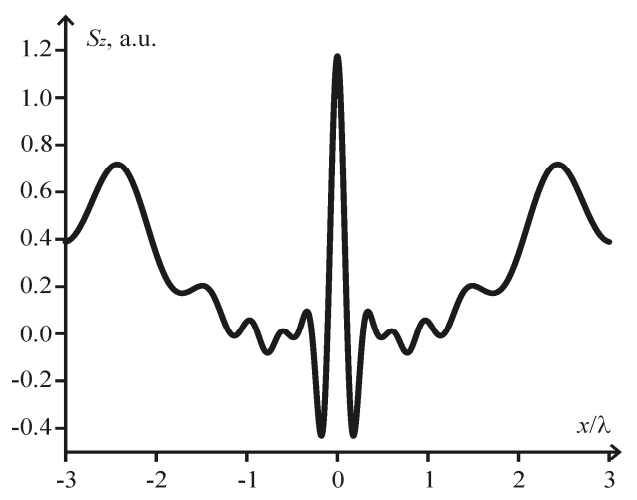

(b)

Figure 6. Time-averaged (500 values of last 10 periods) density of energy (intensity $\left.\left|E_{y}\right|^{2}\right)$ (a) and z-axis projection of power flow (Poynting vector) (b) in plane located at a distance of $\lambda / 50$ beyond the lens.

The fact that the focal spot is smaller in size than the diffraction limit $(0.44 \lambda$ in $2 \mathrm{D}$ case $)$ can be explained by the existence of surface waves. In order to prove this, let us consider the amplitude spectrum in a plane located at distance $\lambda / 50$ beyond the lens. Shown in figure 7 are the normalized spectra of the amplitudes $E_{y}$ in a plane located at distance $2 \lambda / 50$ (curve $a$ ) and $\lambda / 50$ (curve $b$ ) beyond the lens's rear surface. It is seen from figure 7 that the spectrum has non-zero values at $|\xi| \geq 1$, which proves the existence of the evanescent waves. Curve $b$ is seen to be slightly above curve $a$, because closer to the lens the evanescent waves have a stronger impact onto the focal spot (because ampli- 
tude of surface wave drops exponentially and therefore the larger is the distance from the surface, the weaker is the amplitude of the surface wave).

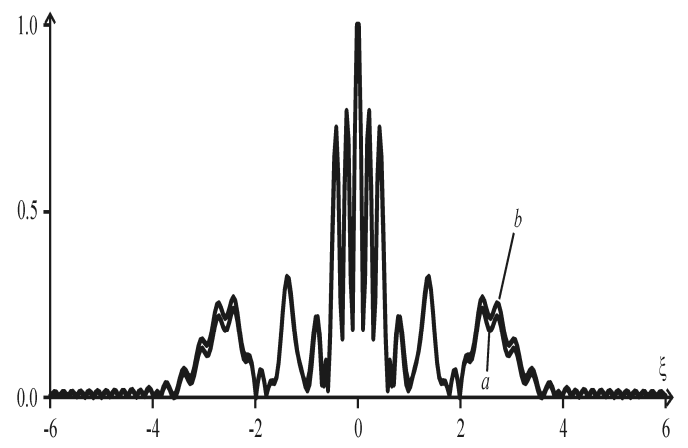

Figure 7. Spectra of the amplitude $E_{y}$ in a plane at distance (a) $2 \lambda / 50$ and (b) $\lambda / 50$ beyond the lens.

To prove that the grating allows the contribution of the evanescent waves into the focal spot to be increased, we also calculated the angular spectrum of plane waves at a distance of $\lambda / 50$ from the grating. The other simulation parameters were the same. The grating period was $300 \mathrm{~nm}$, the grating depth was $300 \mathrm{~nm}$, and the grating fill-factor was $50 \%$. In this case, the focal spot width was also equal to FWHM = $0.114 \lambda$. Shown in figure 8 is the resulting angular spectrum.

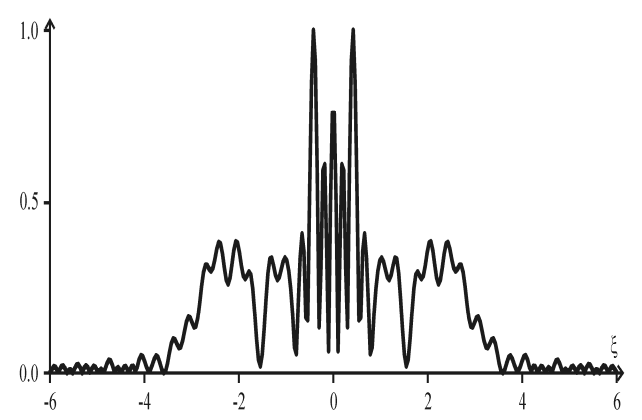

Figure 8. The angular spectrum of plane waves of amplitude $E_{y}$ at distance $\lambda / 50$ from the grating.

It is seen from figure 8 that the main contribution to the focal spot is not from the normally incident plane wave, but from two tilted plane waves propagating at an angle arc$\sin (0.4) \approx 23^{\circ}$ with the optical axis. Besides, the contribution of the surface waves is also increasing as suggested by scarcely noticeable maxima in the region $|\xi| \geq 1$.

The measurements at distance $\lambda$ from the grating show that 10 percent of the total light energy incident onto the lens front surface is directed to the 0 -th diffraction order.

\section{Focusing Light with a Binary HS-Lens}

Let us consider a 2D binary lens with the zone sizes such as to produce the effective refractive index described by equation (8). Such a binary lens will be referred to as a binary HS lens.

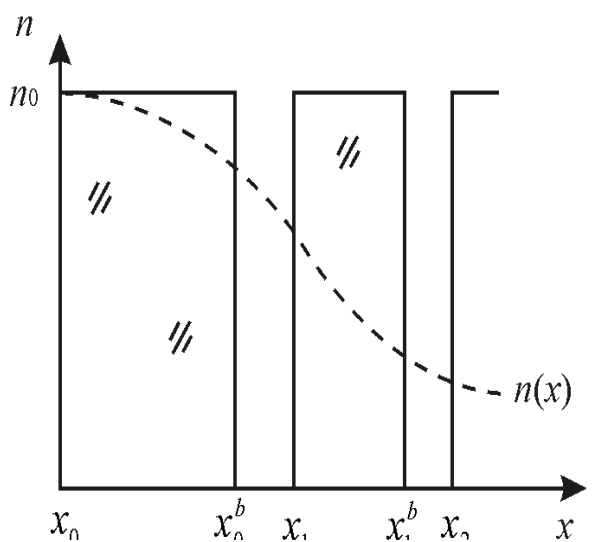

Figure 9. Refractive index profiles of the gradient-index HS lens (dashed line) and the corresponding binary HS lens (solid line). Shaded area denotes silicon..

The index profiles of the gradient-index HS lens and the corresponding binary HS lens are shown in figure 9. The radius of the binary lens is broken down into the intervals $\left[x_{m}\right.$, $\left.x_{m+1}\right], m=0,1,2, \ldots, M$, with a point $x_{m}<x_{m}{ }^{b}<x_{m+1}$ found in each interval, such that on the interval $\left[x_{m}, x_{m}{ }^{b}\right]$ the lens material is silicon, and on the interval $\left[x_{m}{ }^{b}, x_{m+1}\right]-$ air. The point $x_{m}{ }^{b}$ is chosen such as to approximate the refractive index of equation (8):

$$
\int_{x_{m}}^{x_{m+1}} n(x) \mathrm{d} x=n_{0}\left(x_{m}^{b}-x_{m}\right)+1 \cdot\left(x_{m+1}-x_{m}^{b}\right)
$$

Note that for the index distribution in equation (8), the left side can be computed analytically, whereas from equation (14) we can derive an explicit expression for $x_{m}{ }^{b}$ :

$$
\begin{aligned}
& x_{m}^{b}=\frac{n_{0} x_{m}-x_{m+1}}{n_{0}-1}+\frac{1}{n_{0}-1} \frac{4 n_{0} L}{\pi} \times \\
& \times\left\{\arctan \left[\exp \left(\frac{\pi x_{m+1}}{2 L}\right)\right]-\arctan \left[\exp \left(\frac{\pi x_{m}}{2 L}\right)\right]\right\} .
\end{aligned}
$$

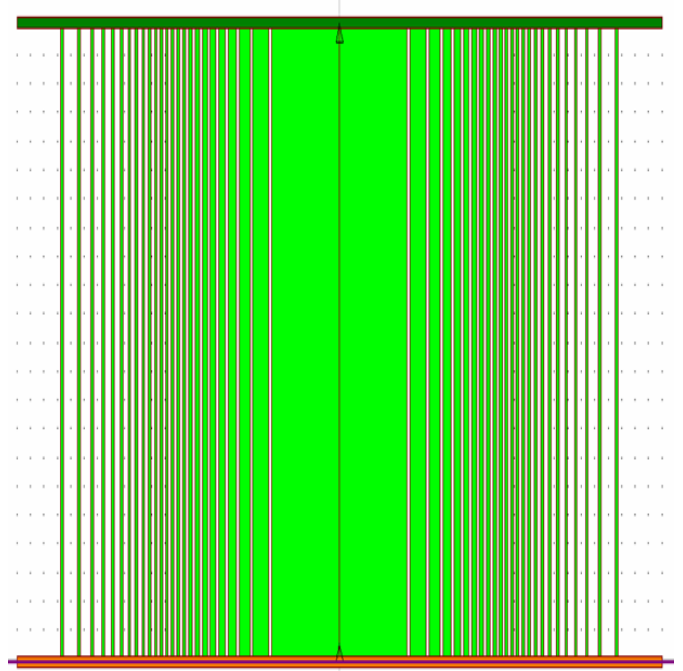

(a) 


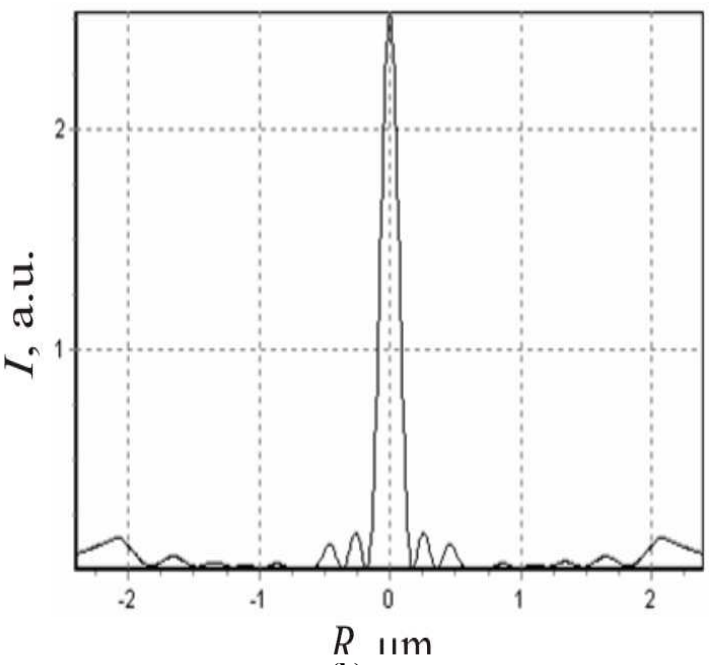

(b)

Figure 10. (a) Binary HS lens $(n=3.47)$, horizontal dimension, $2 R=4.8$ $\mu \mathrm{m}$, vertical $-L=2 \mu \mathrm{m}$, minimal groove- $20 \mathrm{~nm}$ (filled with air). Light propagates vertically. (b) Transverse intensity distribution $|E y|^{2}$ at the lens output (10 nm apart).

Shown in figure 10(a) is a binary HS lens designed using equation (14) on the basis of the lens in figure 4(a), and in figure 10 (b) is the intensity distribution in the focal plane at the lens output. The focal spot width in figure 10(b) is $\mathrm{FWHM}=159 \mathrm{~nm}=0.102 \lambda$. This value is $17 \%$ smaller than the focal spot size in figure 4(b) and $20 \%$ smaller than the diffraction limit of equation (10). Thus, we can infer that in compliance with figure 2 , the focal spot at the binary HS lens output (figure $10(\mathrm{~b})$ ) is formed with the $20 \%$ contribution of second-type surface waves $\left(k_{x}>n k\right)$.

\section{Imaging with a Double HS-Lens}

Shown in figure 11(a) is an instantaneous pattern of the electric vector of the light wave in the HS-lens (made of silicon, $n=3.47$, width $2 R=6 \mu \mathrm{m}$, length $2 L=4.92 \mu \mathrm{m}$ ) from two point sources (each is a Gaussian beam with $50-\mathrm{nm}$ waist radius and free space wavelength of $\lambda=1 \mu \mathrm{m}$ ) separated by $300-\mathrm{nm}$ distance and located $10-\mathrm{nm}$ away from the lens input surface. The simulation was conducted by the FDTD-method with the space sampling of $\lambda / 100$ and the simulation time of $200 \lambda / c, c$ being the speed of light in a vacuum. Figure 11(b) shows the time-averaged distribution of the Poynting vector's projection onto the optical axis $z$, calculated beyond the HS-lens output surface at a distance of $10 \mathrm{~nm}$. It is seen from figure 11(b) that the two sources are resolved and the superresolution value is $0.3 \lambda$. Although the image of the point source has a width of FWHM $=0.12 \lambda$, the two point sources are surely resolved only when separated by a distance of $0.3 \lambda$. This is because the images of these two sources interfere with each other.

Note that such a gradient waveguide can be implemented as a photonic crystal device [35].

Below, we consider the power flux (z-projection of Poynting vector) rather than the intensity. In this case, two light sources can be resolved even if they are closer to each other. Figure 12 shows that two sources separated by a distance of $0.25 \lambda$ are surely resolved in terms of the power flux and scarcely resolved in terms of the intensity. When separated by a distance of $0.15 \lambda$, the sources can be resolved only in terms of the power flux, as it can be seen from figure 13 . When separated by a distance of $0.12 \lambda$, the light sources can be resolved neither via the power flux nor via the intensity (figure 14).

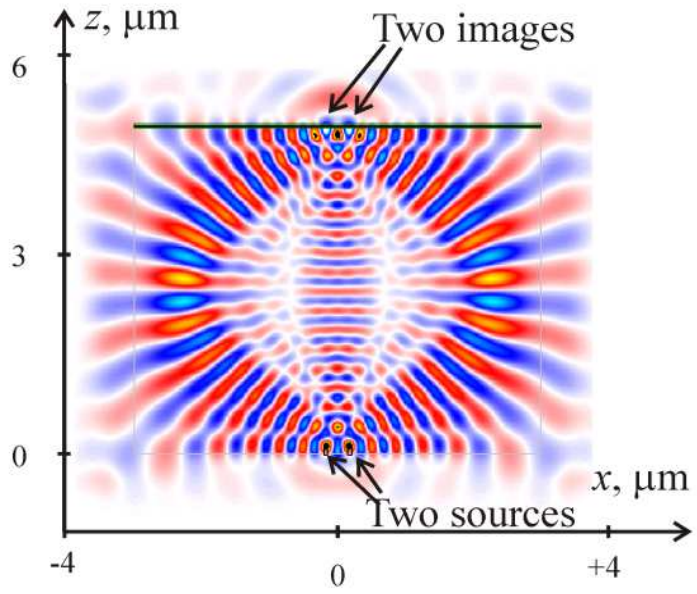

(a)

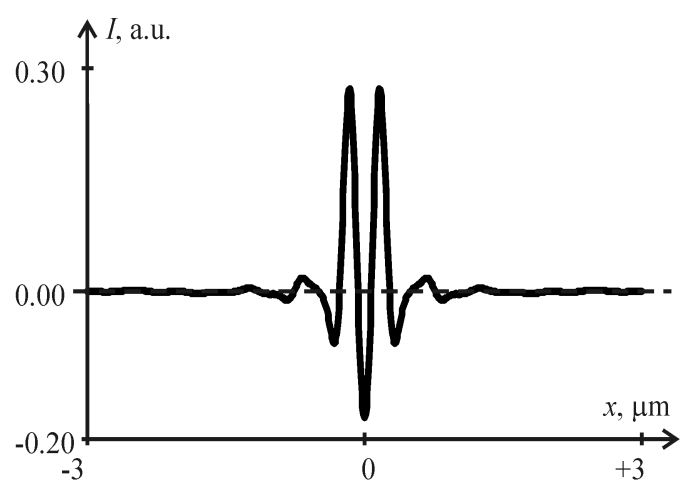

(b)

Figure 11. (a) Instantaneous pattern of the electric vector amplitude of the TE-wave in the HS-lens from two point sources (each is 50-nm wide), separated by 300-nm distance and located 10-nmaway from the input plane; (b) time-averaged distribution of Poynting vector's projection onto the optical axis calculated 10-nm away from the waveguide output plane (top horizontal line), the intensity is shown in arbitrary units.

In the case of the TE-polarization, the power flux $(\mathbf{S}=\mathbf{E} \times \mathbf{H})$ can be measured in the following way. It is related with the intensity as follows:

$$
S_{z}=E_{y} H_{x}^{*}=E_{y} \frac{i}{\omega \mu_{0} \mu} \frac{\partial E_{y}^{*}}{\partial z}=\frac{i}{2 \omega \mu_{0} \mu} \frac{\partial I}{\partial z},
$$

where $\omega$ is the frequency, $\mu$ is the magnetic permittivity, $I$ $=E_{y}{ }^{2}$ is the intensity. Therefore, the power flux can be measured as the difference of the intensities in two near parallel planes. 


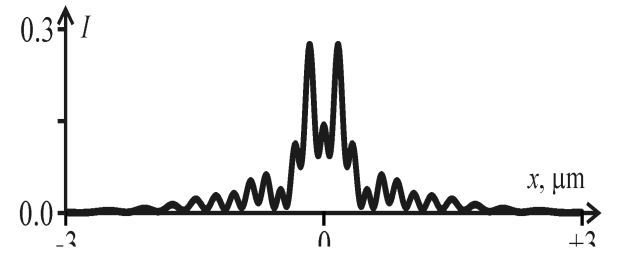

(a)

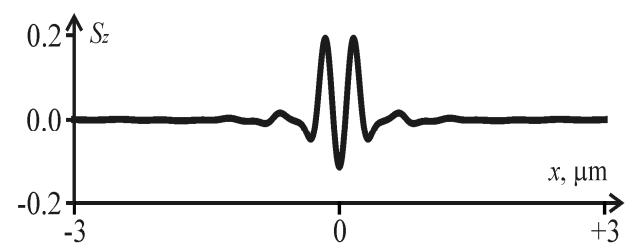

(b)

Figure 12. Time-averaged intensity (a) and power flow (b) calculated at a distance of 10-nm from secant waveguide output plane for two light sources with center-to-center distance of $250 \mathrm{~nm}$.

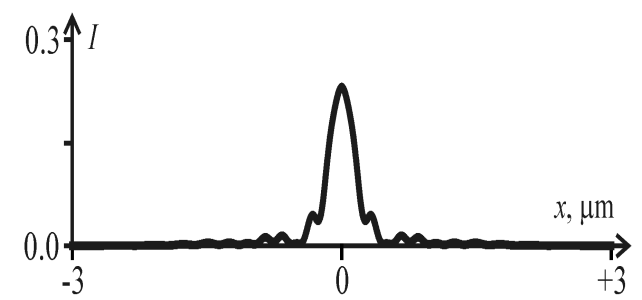

(a)

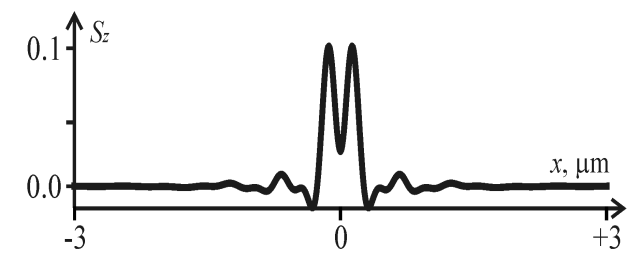

(b)

Figure 13. (a) Time-averaged intensity and (b) power flux calculated at a distance of 10-nm from the secant waveguide output plane for two light sources with the center-to-center distance of $150 \mathrm{~nm}$.

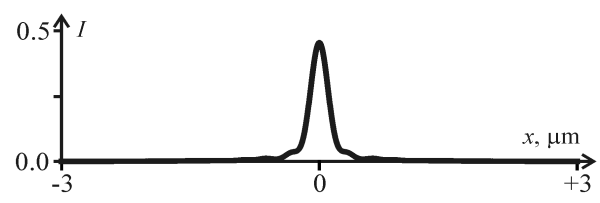

(a)

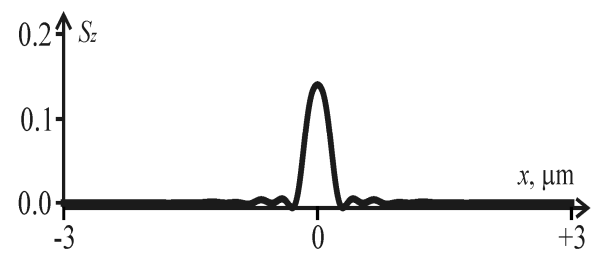

(b)

Figure 14. (a) Time-averaged intensity and (b) power flux calculated at a distance of 10-nm from the secant waveguide output plane for two light sources with the center-to-center distance of $120 \mathrm{~nm}$.

\section{Conclusions}

We have obtained the following results:

The integral representation of the amplitude of a TE-wave propagating from a point source and behind a plane-parallel plate is derived as a sum of three terms that describe three types of light waves: propagating waves and (inhomogeneous evanescent) surface waves of the first and second types;

The comparison of the NAs of the near-field refractive lenses (SIL, NAIL) and a planar HS lens has shown them to be similar, with the difference for silicon being as small as $5 \%$;

Simulation using the FullWAVE software has shown that by combining the gradient-index HS lens with a subwavelength diffraction grating or replacing the lens with its binary analog the focal spot size can, respectively, be reduced by $10 \%$ and $20 \%$ when compared with the diffraction-limited size in the medium. This implies that the second-type surface waves respectively provide a 10 percent and 20 percent contribution to the subwavelength focal spot at the HS lens output.

The HS-lens with a length of $2 L$ allows one to resolve two point sources with the center-to-center distance of $0.15 \lambda$ via measuring the power flux.

A planar binary silicon microlens to generate near its surface a focal spot of size FWHM $=0.102 \lambda$ has been designed.

\section{Acknowledgements}

This work was financially supported by The Ministry of education and science of Russian Federation (project 8027), RF Presidential grants for support of leading scientific schools (NSh-4128.2012.9), a Young Candidate of Science grant (MK-3912.2012.2) and RFBR grants (12-07-00269, 12-07-31117, 13-07-97008).

\section{References}

[1] K. Huang, P. Shi, X. Kang, X. Zhang, and Y. Li, "Design of DOE for generating a needle of a strong longitudinally polarized field," Opt. Lett., vol. 35, pp. 965-967, 2010.

[2] E.T.F. Rogers, J. Lindbery, T. Roy, S. Savo, J.E. Chad, M.R. Dennis, N.I. Zheludev, "A super-oscilatory lens optical microscope for subwavelengh imaging," Nat. Mater., vol. 11, pp. 432-435, 2012.

[3] J.Y. Lee, B.H. Hong, W.Y. Kim, S.K. Min, Y. Kim, M.V. Jouravlev, R. Bose, K.S. Kim, I.-C. Hwang, L.J. Kaufman, C.W. Wong, P. Kim, and K.S. Kim, "Near-field focusing and magnification through self-assembled nanoscale spherical lenses," Nature, vol. 460, pp. 498-501, 2009.

[4] D.J. Godstein, "Resolution in light microscopy studied by computer simulations,” J. Microsc. 166, pp. 185-197, 1992.

[5] L. Novotny, and B. Hecht, Principles of Nano-Optics, Cambridge University Press, 2006.

[6] A. Bouhelier, J. Renger, M.R. Beversluis, and L. Novotny, 
"Plasmon-coupled tip-enhanced near-field optical microscopy,” J. Microsc., vol. 210, pp. 220-224, 2003.

[7] K. Karrai, and X. Lorenz, "Enhanced reflectivity contrast in confocal solid immersion lens microscopy," Appl. Phys. Lett., vol. 77, pp. 3459-3461, 2000.

[8] S.B. Ippolito , B.B. Goldberg, and M.S. Unlu, "High spatial resolution subsurface microscopy," Appl. Phys. Lett., vol. 78, pp. 4071-4073, 2001.

[9] F.H. Koklu, S.B. Ippolito, B.B. Goldberg, and M.S. Ünlü, "Subsurface microscopy of integrated circuits with angular spectrum and polarization control," Opt. Lett., vol. 34, pp. 1261-1263, 2009.

[10] D. Karabacak, K. Ekinci, C. Gan, G. Gbur, M. Ünlü, S. Ippolito, B. Goldberg, and P. Carney, "Diffraction of evanescent waves and nanomechanical displacement detection," Opt. Lett., vol. 32, pp. 1881-1883, 2007.

[11] D.R. Mason, M.V. Jouravlev, and K.S. Kim, "Enhanced resolution beyond the Abbe diffraction limit with wavelength-scale solid immersion lenses," Opt. Lett., vol. 35, pp. 2007-2009, 2010.

[12] K. Huang, Y. Li Realization of a subwavelength focused spot without a longitudinal field component in a solid immersion lens-based system Opt. Lett., vol. 36, pp. 3536-3538, 2011.

[13] D. Mc Closkey, J.J. Wang, and J.F. Donegan, "Low divergence photonic nanojets from Si3N4 microdisks," Opt. Express, vol. 20, pp. 128-140, 2011.

[14] A. Di Falco, S.C. Kehr, and U. Leonhardt, "Luneberg lens in silicon photonics,” Opt. Express, vol. 19, pp. 5156-5162, 2011.

[15] T. Zentgrat, Y. Liu, M.N. Mikkelsen, J. Valentine, and X. Zhang, "Plasmonic Luneberg and Eaton lenses," Nat. Nanotechn. 6, pp. 151-155, 2011

[16] H. Wu, L.Y. Jiang, W. Jia, and X.Y. Li, "Imaging properties of an annular phtonic crystal slab for both TM-polarization and TE-polarization,” J. Opt., vol. 13, pp. 095103, 2011.

[17] X. Zhang, and Z. Liu, "Superlenses to onercome the diffraction limit," Nat. Mater., vol. 7, pp. 435-441, 2008.

[18] J. Rho, Z. Ye, Y. Xiong, X. Yin, Z. Liu, H. Choi, G. Bartal, and $\mathrm{X}$. Zhang, "Spherical hyperlens for two-dimensional sub-diffractional imaging at visible frequencies," Nat. Commun., vol. 1, pp. 143, 2010.

[19] W. Chen, R.L. Nelson, and Q. Zhan, "Efficient miniature circular polarization analyzer design using hybrid spiral plasmonic lens," Opt. Lett., vol. 37, pp. 1442-1445, 2012.

[20] S. Huang, H. Wang, K. Ding, and L. Tsang, "Subwavelength imaging enhancement through a tree-dimmensional plasmon superlens with rough surface," Opt. Lett., vol. 37, pp. 1295-1297, 2012.
[21] J. Martin, J. Proust, D. Gerard, J. Bijcon, and J. Plain, "Intense Bessel-like beams arising from pyramid-shaped microtips," Opt. Lett., vol. 37, pp. 1274-1276, 2012.

[22] F. De Angelis, F. Gentile, F. Mecarini et al, "Breaking the diffusion limit with super-hydrophobic delivery of molecules to plasmonic nanofocusing SERS structures," Nat. Nanophot., vol. 5, pp. 682-687, 2011.

[23] F.M. Huang, N. Zheludev, Y. Chen, and F.J. Garcia de Abajo, "Focusing of light by a nanohole array," Appl. Phys. Lett., vol. 90, pp. 091119, 2007.

[24] V.V. Kotlyar, S.S. Stafeev, L. O'Faolain, and V.A. Soifer, "Tight focusing with a binary microaxicon," Opt. Lett., vol. 36, pp. 3100-3102, 2011.

[25] X. Li, Y. Cao, and M. Gu, "Superresolution focal-volume induced 3.0 Tbytes/disk capacity by focusing a radially polarized beam," Opt. Lett., vol. 36, pp. 2510-2512, 2011.

[26] A. Vainrub, O. Pustovyy, and V. Vodyanov, "Resolution of 90 $\mathrm{nm}(\lambda / 5)$ in an optical transmission microscope with an annular condenser," Opt. Lett., vol. 31, pp. 2855-2857, 2006.

[27] Y. Kuznetsova, A. Neumann, S.R.J. Brueck, "Solid-immersion imaging interferometric nanoscopy to the limits of available frequency space," J. Opt. Soc. Am. A, vol. 29, pp. 772-781, 2012.

[28] J.W. Goodman, "Introduction to Fourier Optics", Roberts and Company Publishers, 491 p., 2005.

[29] Radio optics, M.: Sov. Radio, 304 p., 1975 (in Russian).

[30] M. Born, E. Wolf, "Principles of Optics", 6 ed., Pergamon, 1986.

[31] Y.R. Triandaphilov, and V.V. Kotlyar, "Photonic Crystal Mikaelian Lens," Optical Memory and Neural Networks (Information Optics), vol. 17, pp. 1-7, 2008.

[32] V.V. Kotlyar, A.A. Kovalev, and V.A. Soifer, "Subwavelength Focusing with a Mikaelian Planar Lens," Optical Memory and Neural Networks (Information Optics), vol. 19, pp. 273-278, 2010.

[33] C. Handmer, S. De Sterke, R. McPhedran, L. Botten, M. Steel, and A. Rahmani, "Blazing evanescent gtrating orders: a spectral approach to beating the Rayleigh limit," Opt. Lett., vol. 35, pp. 2846-2848, 2010.

[34] S. Thongrattanasiri, N.A. Kuhta, M.D. Escarra, A.J. Hoffman, C.F. Gmachl, and V.A. Podolskiy, "Analytical technique for subwavelength far field imaging," App. Phys. Lett., vol. 97, pp. 101103, 2010.

[35] M.I. Kotlyar, Y.R. Triandafilov, A.A. Kovalev, V.A. Soifer, M.V. Kotlyar, and L. O'Faolain, "Photonic crystal lens for coupling two waveguides," Appl. Opt., vol. 48, pp. 3722-3730, 2009. 